\title{
Optimum Conditions for EOR Using Nanofluids Subjected to EM Waves
}

\author{
Muhammad Kashif ${ }^{1, \text { a }}$, Poppy Puspitasari ${ }^{2}$ \\ ${ }^{1}$ Physics Department, University of Agriculture, Faisalabad, Pakistan \\ ${ }^{2}$ Department of Mechanical Engineering, Engineering Faculty, Universitas Negeri Malang, Malang St. No .5 Malang, \\ 65140, East Java, Indonesia \\ Corresponding author: *kashifmughal79@gmail.com
}

\begin{abstract}
Today's major challenge for oil industry is to improve the oil recovery from the reservoir. Various enhanced oil recovery (EOR) methods have been applied in the field and the steam injection is one of the most favourable methods. The deep reservoir will result in failure of this method due to excessive heat dissipation. In this situation, generating and injecting steam may be uneconomical due to the tremendous reduction of the recovery. Some methods using nanotechnology have been introduced and elaborated. However, we propose the electromagnetic (EM) method as an alternative due to its long range transmission of the transverse waves. These EM waves, coupled with some nanoparticles (NP), can modify the surface energy. We propose an optimum conditions based on some parameters namely, frequency, flux density, space charge density and skin depth, employing Maxwell and Helmholtz equations which interact with some magnetic and dielectric nanoparticles. A newly-designed EM antenna with a very high flux density is the model for this specific purpose. The electrical energy from the antenna transfers the waves to the dielectric and resistive nanoparticles, which is then transferred to the fluid with high capillary force. This results in lower surface tension which reduces the oil viscosity. In order to investigate the transport phenomena of the nanoparticles in porous medium, we applied Darcy's law. Our preliminary study for scale model simulations showed that at a frequency of $0.125 \mathrm{~Hz}$, the electric field of the curve antenna with magnetic feeders was $4280 \%$ higher compared to the one without magnetic feeders, At a frequency of $0.125 \mathrm{~Hz}$, the magnetic field of the curve antenna with magnetic feeders was $3677 \%$ higher in comparison with the one without magnetic feeders. With the increasing frequency from $0.125 \mathrm{~Hz}$ to $9 \mathrm{~Hz}$, the electric field and magnetic field of the antenna with feeders decreased by $99 \%$. The permeability and porosity of glass beads packed column was $30.58 \mathrm{mD}$ and $25.87 \%$ respectively. It was observed that the cumulative recovery of oil reached $21.11 \%$ by usingZnO nanofluid with electromagnetic waves, $17.23 \%$ by using $\mathrm{ZnO}$ nanofluid without electromagnetic waves, $32.59 \%$ by using iron oxide nanofluid with electromagnetic waves, and $29.68 \%$ by using iron oxide nanofluid without electromagnetic waves. In summary, the use of $\mathrm{ZnO}$ and iron oxide nanoparticles as nanofluids with electromagnetic waves is considered the most effective to use in enhanced oil recovery.

Copyright (C) 2017Journal of Mechanical Engineering Science and Technology

All rights reserved

Keywords: EOR, Nano Fluid, EM Waves
\end{abstract}

\section{Introduction.}

Enhanced oil recovery is important for oil industry;, it is highly needed to know determine the levels of oil recovery. The recovery of oil is classified into three stages.: In the primary stage, the crude oil is taken out from the reservoir by its natural pressure. In some cases, the natural pressure is not enough to push the oil from subsurface or because of the viscosity of oil is very high so that it is not possible to produce oil from the reservoir. The secondary stage is used by pumping water (the most economic material among those other injecting materials) to displace the oil [1]. It is well known that the reservoir consists of pours and channels with a radius of 0.5-50 micrometre, so water sweeps the oil slowly $(30 \mathrm{~cm}$ per day) and many areas are not swept well. [2] As a result, there is a considerable volume of residual oil in the well; and this the volume is between 35 and $65 \%$ [3]. In other words, oil is trapped in the water- in wet system. That trappingIt happens because of the interfacial tension between oil and water is high, so that the viscous/boundary forces are not enough to overcome the large capillary forces at in a narrow pore/ throat within the rock matrix [4]. Therefore, the third stage, i.e. is quite important to extracting the residual oil which is called enhance oil recovery (EOR) or improving oil recovery (IOR), is quite important. 
The chemical, miscible or immiscible, and thermal way are the main methods which are used for enhanced oil recovery (EOR) [5]. The increaseing of oil price excited the researchers to think out of the box, that opening the door to apply a brand new technology which is most much more sophisticated and effective [3].

\section{A. The Function Of Nanofluid In Oil Field}

The subsurface environment in oil reservoir. The natural reservoir consists of silicate clays, iron and aluminum oxides, precipitates of mineral, humid materials and micro organism [6]. $\mathrm{pH}$ is not natural one, high salinity, pressure as well as high temperature. Nanoparticles have been extensively used in oil reservoirs due to its better physical and chemical properties. Coated nanoparticles, can stand against harsh conditions and their charge can be controlled [7] as well as the size can be identified [8].

The coating of nanoparticles can be done in many methods, the most common ones are: by using polymer [9], surfactant [10] and some polar molecules like citric acid [11-14]. In nanoscale, the factors which determine the yield of oil recovery are: pore shape, roughness, water distribution and surfactant film behavior [1]. The use of nanoparticles can destabilise the emulsion required, and control the characteristics and their rheological properties [15]. In oil well environment, nanoparticles are exposed to the following forces: Coulombic, disjoining, Marangoni, capillary, viscous, gravity [4,16-18]

The balance between Van der Waals attractive forces and electrical repulsive forces determines the stability [4]. Van der Waals attractive forces between particles at the same distance are stronger than electrical repulsion so the particles will aggregate with each other. In addition to Van der Waals forces promoting nanoparticles aggregation, another factor which can be responsible of controlling the desorption in nano-scale particle is Brownian motion [19].

In the reservoir environment, nanoparticles reduce the interfacial tension between water wet system and crude oil, by creating emulsion phase [4]. These particles simultaneously increase the viscosity of aqueous phase. The viscosity, increases as the concentration of nanoparticles increases [19], playings an important role to improve the displacement of oil from the pore. Suleimanov et al. investigated the effects of the presence of surfactant in the formation of nanoparticles in an aqueous phase. It was found that the surface tension decreased by more than $69 \%$ by adding nanoparticles to the aqueous phase [20]. The stability of adsorption process with nanoparticles was better in comparison with surfactant alone; the adsorption values was 14.5-18.5fold better. Furthermore, adding nanoparticles to can turn the flow from Newtonian into nonNewtonian. In light of the main focus of this article, it was concluded that nanoparticles were more efficient in enhancing oil recovery in both heterogeneous and homogeneous pore medium in comparison with water and surfactant [20].

\section{Methods.}

\section{A. Antenna Design}

We proposed a new improved antenna design for enhanced oil recovery application. In this new design, the antenna was fed in two ways: with excitation at the antenna end and with magnetic feeders (ferrite core with toroidal wire) connected in series. This antenna consists of two conductors and one dielectric material. Two slots were made on the outer conductor at a distance of $\lambda / 4$ from the each end. The length of the antenna is equal to a full wavelength, which is a combination of two $\lambda / 2$ antennas. The slots act as the excitation source for these two antennas. A square wave form was used to excite the dipole antennas. This wave form was applied to transfer the maximum energy to the subsurface sea floor since this wave form has the maximum energy due to the current flowing all the time at its peak values [21]. The only drawback of this wave form is that all energy was transferred simultaneouslyin the first harmonic region. If a square wave with multiple frequencies is used for depth migration and inversion analysis, then it will show very promising results. The length of the antenna is related to the frequency applied to operate the antenna. The types of antenna according to the wavelength are full wavelength, half wavelength and quarter wavelength. In sea bed logging, a half wavelength dipole antenna is used due to its high efficiency over other wavelengths of antennas. The wavelength of the electromagnetic waves transmitted by the antenna is given [22] in equation (1). 


$$
\begin{aligned}
& \lambda=2 \pi \sqrt{\frac{2}{\mu \omega \sigma}} \\
& \lambda=\text { wavelength of electromagnetic waves transmitted by antenna } \\
& \mu=\text { permeability } \\
& \sigma=\text { conductivity of the medium } \\
& \omega=\text { angular frequency }
\end{aligned}
$$

The wavelength of electromagnetic waves transmitted by an antenna can be calculated with the equation (1). After the wavelength was calculated, the length of the straight dipole antenna can be calculated with the help of the equation (2) [23].

$$
\begin{aligned}
& \frac{\lambda}{2}=L \\
& L=\pi r
\end{aligned}
$$

The length of the new dipole antenna was calculated with the help of equation (3), where $\mathrm{L}$ is the length of the dipole and $\mathrm{r}$ is the curvature radius of the new dipole antenna.

\section{B. Nanoparticles Synthesis}

$\mathrm{Fe}_{2} \mathrm{O}_{3}$ nanoparticles were prepared by sol-gel method based on Iron nitrate $\left[\left(\mathrm{Fe}\left(\mathrm{NO}_{3}\right) 3.9 \mathrm{H}_{2} \mathrm{O}\right)\right]$ nitric acid $\left(\mathrm{HNO}_{3}\right)$ materials. All nitrates used in the synthesis of $\mathrm{Fe} 2 \mathrm{O} 3$ were dissolved in the aqueous solution of nitric acid $\mathrm{HNO}_{3}$. The solution was stirred at 250 r.p.m for 7 days. The solution was allowed to combust on the hot plate stirrer. After 30 minutes, the temperature was gradually raised until it reached $70 \mathrm{oC}$. The gel-like material was dried in the oven at $120 \mathrm{oC}$ for 3 days and the dried powder was grounded for 4 hours. After that, the powderwas annealed for 6 hours to get the required characteristics of metal oxide nanoparticles. The nanopowder was sintered at $300^{\circ} \mathrm{C}, 400^{\circ} \mathrm{C}$ and $500^{\circ} \mathrm{C}$ by using a sintering furnace.

To determine structural characteristics, X-ray diffraction (XRD) measurements were performed, using Bruker D8 advance x-ray diffractometer with $\mathrm{Cu}-\mathrm{k} \alpha$ radiation $(\lambda=1.5406 \AA)$ and an accelerating voltage of $40 \mathrm{kV}$. From the XRD patterns, the crystallite sizes of the iron oxide nanoparticles were calculated using Debye-Scherrer equation (Eq.4)

$$
D=\frac{0.9 \lambda \omega}{\cos \theta}
$$

$\theta=$ Bragg's angle

$\omega=$ Full width at half maximum (FWHM)

$\lambda=$ wavelength of incident radiation

The nanofluids were prepared by direct mixing of the stabilising fluid with the nanoparticles. Firstly, nanoparticles were synthesised in the form of powder. Secondly, this powder was introduced into the stabilising fluid. Best samples of $\mathrm{ZnO}$ and iron oxide nanoparticles were selected for our preliminary study. $\mathrm{ZnO}$ and iron oxide nanoparticles were mixed with sodium dodecyl sulphate (SDS) stabiliser with a concentration of $0.05 \%$. In order to enable thefurther dispersion of $\mathrm{ZnO}$ and iron oxide, they were sonicated in sodium dodecyl sulphate (SDS) for 2 hours. Nanofluids and sodium dodecyl sulphate at the concentration of $0.05 \%$ had higher suspension ability and thus these nanofluids were chosen throughout this project. High stability in suspension is very important and needed to suspend nanofluids for a longer time inside the well and hence will be able to recover more oil.

\section{Enhanced Oil Recovery Setup}

The enhanced oil recovery involving the $\mathrm{ZnO}$ and iron oxide nanofluids was investigated by utilising a synthetic bead packed with a PVC column. The experimental setup included a PVC column, an injection pump, an antenna with magnetic feeders [24-26] and a water tank (as shown in Figure 1). To replicate the reservoir environment, the water tank was filled with salt water having a salinity of $30000 \mathrm{ppm}$ and adensity of $1.006 \mathrm{~g} / \mathrm{cc}$. Electromagnetic waves were produced 


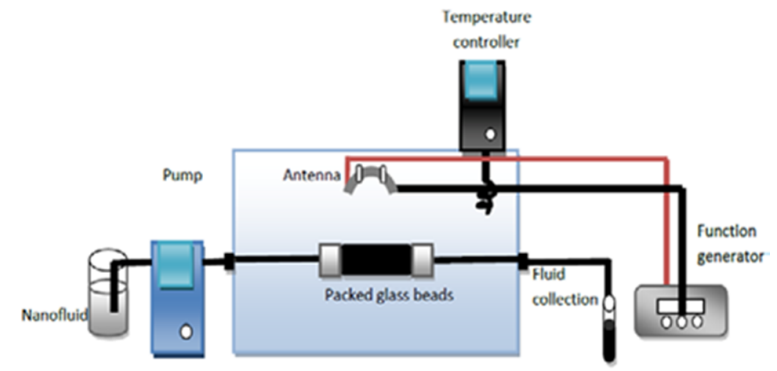

Fig. 1. Schematic diagram experiment setup for $\mathrm{ZnO}$ and iron oxide nanofluids EOR

by connecting the function generator to the curve antenna to supply an AC current at a frequency of $30 \mathrm{MHz}$ and voltage of $10 \mathrm{Vpp}$. The length of the antenna was $25 \mathrm{~cm}$ with two magnetic feeders. The experiment was conducted with the injection of $\mathrm{ZnO}$ and iron oxide nanofluids into the glass beads PVC column, which was exposed to the EM waves. Finally, the percentage of oil recovery was calculated.

\section{Results and discussion.}

\section{A. Scale Modelling of Antenna in offshore environment}

The new EM antenna was simulated in computer simulated software (CST) based on the finite integration method for marine environment. The EM antenna with and without magnetic feeders was simulated at target depth of $1000 \mathrm{~m}$ below seafloor. The simulation of electric field and magnetic field in the proposed antenna was carried out using CST Studio software. Our goal was to compare the electric and magnetic field produced by antenna with and without magnetic feeders. Propagation of electromagnetic (EM) waves travelling in seawater can be predicted by using

Maxwell's equations. If the electromagnetic (EM) wave propagates in the y direction, then it can be described in terms of the electric field strength Ex and the magnetic field strength $\mathrm{Hz}$ [27]. The effect of different low frequencies on the twin collinear antenna with and without magnetic feeders was investigated. In this research, the antenna was modified using magnetic feeders to increase the strength of the electric and magnetic fields. The magnetic feeders were used to excite the TM components such as $\mathrm{H} \varphi$, Ez, and E $\rho$. When the magnetic feeders were used on the antenna (conductor), the magnetic flux energy was transferred from the magnetic feeders to the current flowing along the antenna. Higher values of Q gave better efficiency of the power, which was delivered to the antenna current. When toroidal core was excited using AC source, then, due to magnetisation, magnetic field $\mathrm{H}$ was generated. Magnetic field ' $\mathrm{H}$ ' generated around the full loop constraint entire field to the core material, which is according to the Biot and Savarts law. Electric field was concentrated at the middle of the magnetic feeder due to the trapped magnetic field inside the circular loop; it is based on the Maxwell's relations ( $\mathrm{E}$ is perpendicular to the $\mathrm{H}$ field). When a conductor is placed at the point of concentrated electric field, a large amount of current is induced in the wire antenna [28]. Figure 2 (a) and (b) show the E-field response corresponding to different frequencies of curve antenna with and without magnetic feeders. Figure 2 (c) and (d) show the $\mathrm{H}$-field response corresponding to different frequencies of curve antenna with and without magnetic feeders. Fig. 3 shows the comparison of E field response with and without magnetic feeders. It was found that as the frequency increased from $0.125 \mathrm{~Hz}$ to $9 \mathrm{~Hz}$, the Ex and $\mathrm{Hz}$ signal strength decreased up to $99 \%$ due to the attenuation of EM waves. TheEx field response with magnetic feeders was $4280 \%$, whereas the $\mathrm{Hz}$ field response was $3677 \%$. It was observed that a frequency of $0.125 \mathrm{~Hz}$ was suitable for the enhanced oil recovery application due to higher penetration depth. 


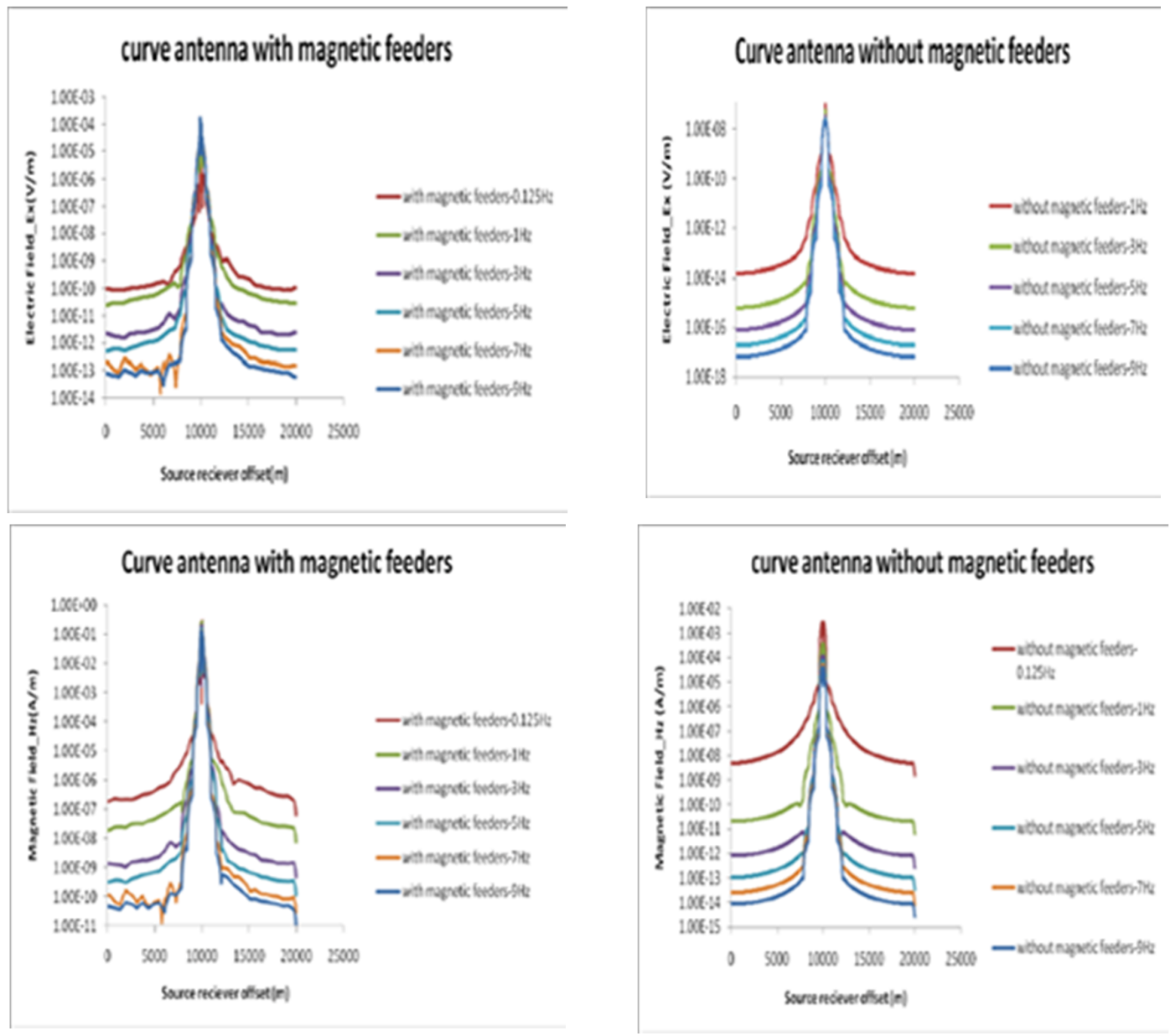

Fig. 2.E-field (a), (b) and H-fields (c), (d) values corresponding to different frequencies of curve antenna with magnetic feeders and without magnetic feeders respectively.

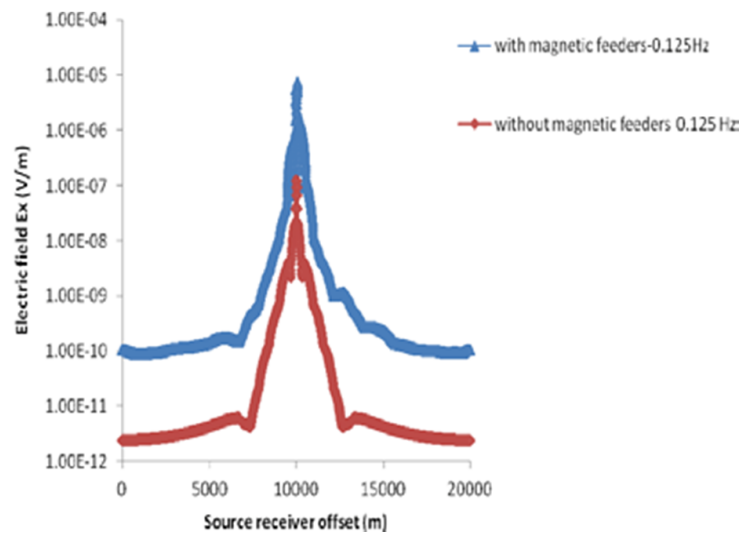

Fig. 3.Comparison of curve antenna with and without magnetic feeders

\section{B. Nanoparticle characterization}

$\mathrm{X}$-ray diffractometer (XRD) was used to get the phase and crystal structure of the prepared samples. X-ray diffraction patterns with plane numbers of iron oxide nanoparticles sintered at $300^{\circ} \mathrm{C}, 400^{\circ} \mathrm{C}$ and $500^{\circ} \mathrm{C}$ temperatures are shown in Figure 4. From Scherer formula, it was calculated that the crystallite size increased from $30.27 \mathrm{~nm}$ to $37.60 \mathrm{~nm}$ by increasing the annealing temperature from $300^{\circ} \mathrm{C}$ to $500^{\circ} \mathrm{C}$. 


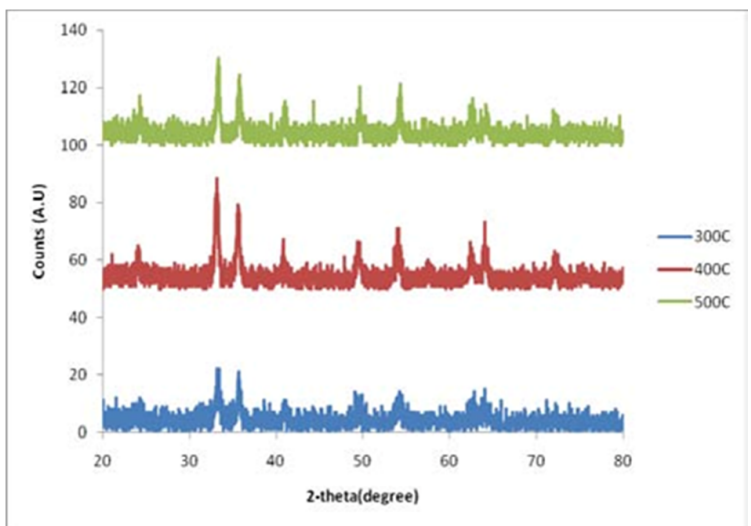

Fig. 4.XRD results of iron oxide nanoparticles sintered at temperature $300^{\circ} \mathrm{C}, 400^{\circ} \mathrm{C}$ and $500^{\circ} \mathrm{C}$.
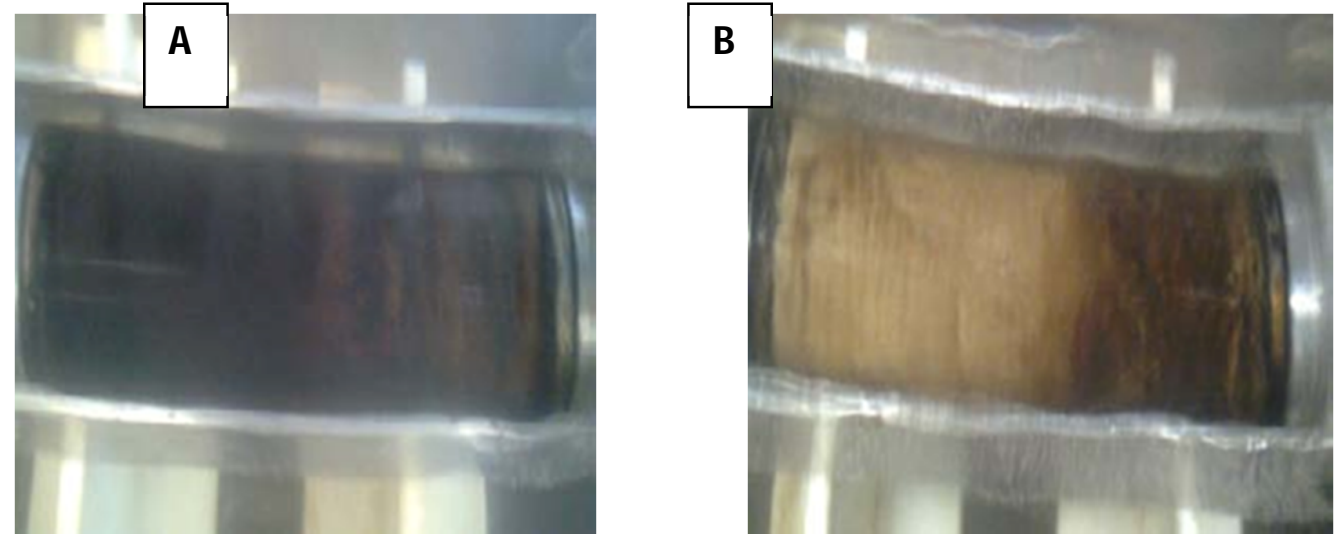

Fig. 5.Glass beads column (a) saturated with oil (b) after nanofluid injection

\section{Enhanced Oil Recovery by Using Nanofluids Injection and Electromagnetic Waves}

$\mathrm{ZnO}$ nanoparticles were synthesized with a microwave sintering at 30 minutes. The PVC column packed with glass beads was saturated with a brine with salinity of 30000ppm until a constant pressure was achieved. The porosity and permeability of the sample is presented in the Table.2. When the pressure was stabilised (constant pressure), crude oil was injected into the glass beads PVC column. Crude oil replaced the brine in the glass beads and the brine was collected from a cylinder. The volume of the brine represented the volume of the crude oil in the glass beads. This volume of the oil in the glass beads represented the amount of original oil in place (OOIP). Water flooding was carried out by injecting brine a second time into the glass beads PVC column until it was confirmed that there was no more oil dropping from the outlet tube. Water flooding replaced some amount of OOIP and the volume of oil displaced from the glass beads, which represented the volume of oil recovered after water flooding. The amount of the oil remained in the glass beads termed as residual oil.

$\mathrm{ZnO}$ nanofluids were injected into the glass beads column following the water flooding to simulate EOR process. During $\mathrm{ZnO}$ nanofluid injection glass beads PVC column was exposed to electromagnetic waves emitted from antenna. The same procedure was applied for iron oxide nanofluid injection. Figure 5 (a) and (b) show the glass beads column after being saturated with oil and nanofluid injection. During nanofluid injection, the recovered oil was collected and the experiment was stopped when it was confirmed that there was no more oil dropping from the outlet tube. The $\mathrm{ZnO}$ nanofluid injection yielded $21.11 \%$ and the oil recovered from ROIP was $17.23 \%$. The Iron oxide nanofluid injection yielded was $32.59 \%$ and oil recovered from ROIP was $29.68 \%$. Table.1 tabulates the OOIP and the percentages of oil recovery by nanofluids injection. Figure 6 represents the oil recovery (\%OOIP) from pore volume of the injected fluid in glass beads packed sample at displacement by nanofluid slug with and without electromagnetic waves. 


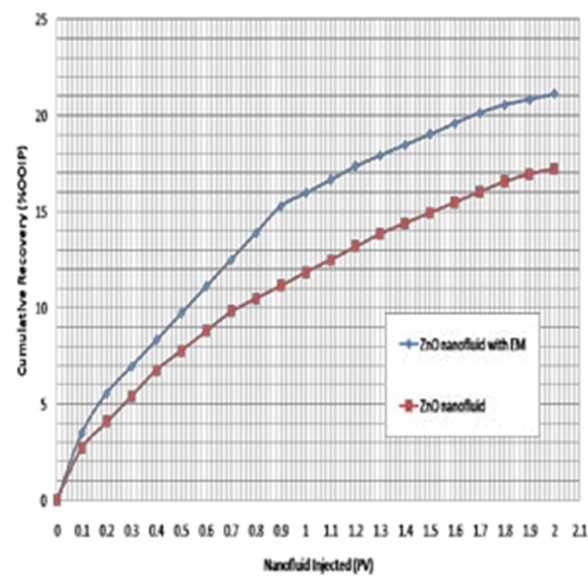

(A)

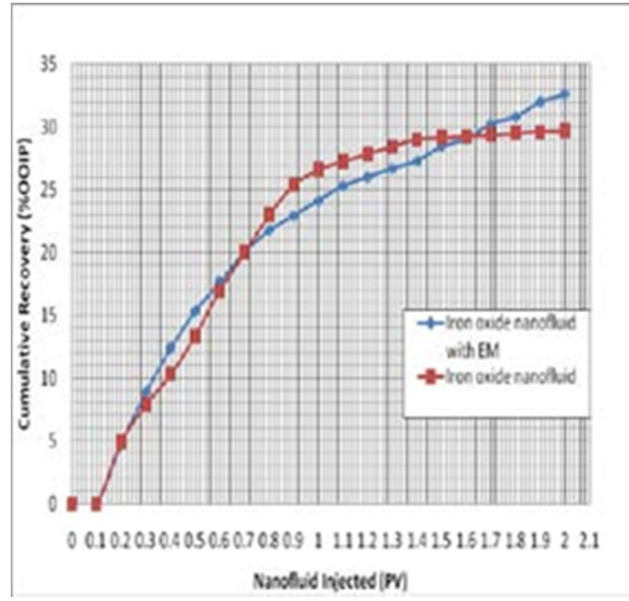

(B)

Fig. 6. Oil recovery (\%OOIP) from pore volume of the injected fluid in glass beads packed sample at displacement by (a) $\mathrm{ZnO}$ nanofluid (b) Iron oxide nanofluid slug with and without electromagnetic waves.

Table 1. Original oil in place (OOIP) and the percentages of oil recovery by $\mathrm{ZnO}$, Iron oxide nanofluids injection

\begin{tabular}{ccccc}
\hline & \multicolumn{2}{c}{ ZnO nanofluid } & \multicolumn{2}{c}{ Iron Oxide nanofluid } \\
\cline { 2 - 5 } & $\begin{array}{c}\text { NF } \\
\text { With EM }\end{array}$ & $\begin{array}{c}\text { NF } \\
\text { Without EM }\end{array}$ & $\begin{array}{c}\text { NF } \\
\text { with EM }\end{array}$ & $\begin{array}{c}\text { NF } \\
\text { Without EM }\end{array}$ \\
\hline Porosity (\%) & 25.18 & 25.87 & 30.17 & 27.57 \\
\hline Permeability (mD) & 29.71 & 30.58 & 77.60 & 82.05 \\
\hline OOIP (ml) & 72 & 74 & 85 & 82.72 \\
\hline ROIP(ml) & 16 & 13.4 & 29 & 32.72 \\
\hline $\begin{array}{c}\text { Vol. of oil recovered } \\
\text { duringWF(ml) }\end{array}$ & 56 & 60.6 & 56 & $29.68 \%$ \\
\hline Recovery (OOIP) & $21.11 \%$ & $17.23 \%$ & $32.59 \%$ & \\
\hline
\end{tabular}

As shown in Table 1,the production rate of oil displaced by the nanofluid with electromagnetic increased for $22.51 \%$ in comparison with the nanofluid. Iron oxide nanofluid with EM waves gave $54 \%$ more oil recovery as compared to $\mathrm{ZnO}$ nanofluid with EM waves.

\section{Conclusions}

A new antenna with and without magnetic feeders was designed and simulated in CST EM studio. The scale model simulation showed that at a frequency of $0.125 \mathrm{~Hz}$, the electric field of the curve antenna with magnetic feeders was $42.80 \%$ higher compared to the one without magnetic feeders. Moreover, the magnetic field of the curve antenna with magnetic feeders was $36.77 \%$ higher in comparison with the one without magnetic feeders. With the increasing frequency from $0.125 \mathrm{~Hz}$ to $9 \mathrm{~Hz}$, the electric field and magnetic field of the antenna with feeders decreased by $99 \%$. It was observed that the cumulative recovery of oil reached $21.11 \%$ by using $\mathrm{ZnO}$ nanofluid with electromagnetic waves, $17.23 \%$ by using $\mathrm{ZnO}$ nanofluid without electromagnetic waves, $32.59 \%$ by using iron oxide nanofluid with electromagnetic waves, and $29.68 \%$ by using iron oxide nanofluid without electromagnetic waves.

\section{References}

[1] Morrow, N.R.:1990 a."Introduction To Interfacial Phenomena In Oil Recovery" "Interfacial Phenomena In Petroleum Recovery” ed.morrow N.R.,Marcel Dekker Inc., New York,USA

[2] Thomas, S.:2005."Chemical EOR - The past, does it have a future?",SPE Distinguished Lecture Series, SPE108829 
[3] R. L. Mathis: "Reservoir Geology of the Denver Unit- Wasson San Andres Field, Gaines and Yoakum Counties, Texas," Permian Basin SEPM Publication 86-26, 1986, 43-47

[4] A.J.P.Fletcher,SPE,Parr system Pty.Ltd.,and J.P.Davis,University of Bristol "how EOR can be transformed by nanotechnology"

[5] Lake,L., Schmidt,R. and Venuto,P.:1992"A niche for enhanced oil recovery in the 1990;s "Oilfield review, 55-61

[6] McCarthy,J.F., and Zachara,J.M., "Subsurface Transport of Contaminants“, Environmental Since and Technology, Vol.23,No.5,496-502,1989)

[7] H.Yu,SPE,C.Kotsmar,K.Y.Yoon.Ingram,K.P.Johnston,S L.Bryan, and C.Huh, "Transport and retention of Aqueus Dispersions of Paramagnetic Nanoparticles in Reservoir Rocks"

[8] Philipse, A.P.; Van Bruggen, M. P.B.; Pathmamanoharan, C. Magnetic silica dispersions: preparation and stability of surface-modifiedsilica particles with a magnetic core. Langmuir 1994, 10, 92-99.

[9] Ditsch, A.; Laibinis, P. E.; Wang, D. I. C.; Hatton, T.A.

[10]Controlled Clustering and Enhanced Stability of Polymer-Coated Magnetic Nanoparticles. Langmuir 2005, 21 (13), 6006-6018.

[11]Lan, Q.; Liu, C.; Yang, F.; Liu, S.; Xu, J.; Sun, D. Synthesis of bilayer oleic acid-coated Fe3O4 nanoparticles and their application in $\mathrm{pH}$ responsive pickering emulsions. J. Colloid Interface Sci. 2007, 310, 260-269

[12] Campelj, S.; Makovec, D.; Drofenik, M. Preparation and properties of water-based magnetic fluids. J. Phys.: Condens. Matter 2008, 20, 204101-204105.

[13] Sahoo, Y.; Goodarzi, A.; Swihart, M. T.; Ohulchanskyy, T. Y.; Kaur, N.; Furlani, E. P.; Prasad, P. N. Aqueous ferrofluid of magnetite nanoparticles: fluorescence labeling and magnetophoretic control. J. Phys. Chem. B2005, 109, 3879-3885.

[14]Lyon, J. L.; Fleming, D. A.; Stone, M. B.; Schiffer, P.; Williams, M. E. Synthesis of Fe oxide core/ Au shell nanoparticles by iterative hydroxylamine seeding. Nano Lett. 2004, 4, 719-723.

[15]Hui, C.; Shen, C.; Yang, T.; Bao, L.; Tian, J.; Ding, H.; Li, C.; Gao, H.-J. Large-scale Fe3O4 nanoparticles soluble in water synthesized by a facile method. J. Phys. Chem. C 2008, 112, 1133611339.

[16] Thompson, J.,Vasuez, A.,Hill, J.M., and Pereira-Almao,P.," The synthesis and Evaluation of Upscalable Molybdenum based Ultra Dispersed Catalysts : Effect of Temperature on Particle size" Cata.Lett., 123,16-23(2008)

[17]Bresme, F., and Oettel, M.," Nanoparticles at Fluid Interfaces”,J.of Physics-ondensed Matter,19 (41),(2007).

[18]Binks, B.P., and Horozov, T.S.,’Colloidal Particles at Liquid Interfaces:An Introduction',in Colloidal Particles at Liquid Interfaces,Chapt.1Binks.B.P., and Horozov,T.S.,eds.,1-74, Cambridge Univ.press (2006).

[19]Fernandez-Toledano, J.C., Monacho-Jorda,A., Martinez- lopez,F., and Hidaglo-Alvarez,R., "Theory for interactions between Particles in Monolayer" in Colloidal Particles at Liquid Interfaces Binks.B.P., and Horozov,T.S.,eds., 303-301, Cambridge Univ.press (2006).

[20] Elena Rodriguez, Matthew R. Roberts, Haiyang Yu, Chun Huh, and Steven L .Bryant, "Enhanced Migration of Surface-Treated Nanoparticles in Sedimentary Rocks" Annual Technical Conference and Exhibition held in New Orleans, Louisiana, USA, 4-7 October 2009.

[21]B.A. Suleimanov , F.S. Ismailov, E.F. Veliyev Oil Gas Scientific Research Project Institute, SOCAR, Azerbaijan"Nanofluid for enhanced oil recovery" Journal of Petroleum Science and Engineering 78 (2011) 431-437.

[22]R.Mittet and T.S. Pettersen,"Shaping optimal transmitter waveforms for marine CSEM surveys"Geophysics, Vol.73, May-June 2008.

[23]L.Loseth, H.Pedersen, T.S Pettersen, S.Ellingsrud, \& T.Eidesmo, "A scaled experiment for the verification of the SeaBed Logging method". Journal of Applied Geophysics, vol. 64, pp.47-55 2008.

[24] T.A.Milligan, "Modern antenna design ",Second Edition, IEEE Press 2005.

[25]M.N. Akhtar, N. Yahya, N.Nasir, "Novel EM antenna based on Y3Fe5O12 magnetic feeders for improved MVO", Saudi International Electronics, Communications and Photonics Conference, Saudi Arab, (2011).

[26]N. Nasir, N. Yahya, M. Kashif, H. Daud, M. N. Akhter, H. M. Zaid, A. Shafie, L. C.Teng J. Nanosci. Nanotechno, 11(2011)2551-2554. 
[27]N. Yahya, R.M.A. Habashi, Krzysztof Koziol, R. Dunin- Borkowski, M.N. Akhtar, M.

[28] Kashif and M. Hashim, J. Nanosci. Nanotechno, 11(2011)2652-2656.

[29] A. Shaw, A.I. AlShamma', S.R. Wylie and D. Toal ," Experimental Investigations of Electromagnetic Wave Propagation in Seawater"Proceedings of the 36th European Microwave Conference September 2006, Manchester U.K.

[30]M. N. Akhtar, N. Yahya, K. Koziol, and N. Nasir, "Synthesis and characterizations of Ni0.8Zn0.2Fe2O4- MWCNTs composites for their application in sea bed logging," Ceramics International, vol. 37, no. 8, pp. 3237-3245, 2011 